\title{
Stretch reflex oscillations and essential tremor
}

\author{
RODGER J ELBLE, CONSTANCE HIGGINS, CHARLES J MOODY \\ From the Department of Medicine, Division of Neurology, Southern Illinois University School of Medicine, \\ Springfield, Illinois, USA
}

SUMMARY Using a computer-controlled torque motor and manipulandum, $50 \mathrm{~ms}$ torque pulses and 70 second trains of binary pseudorandom torque disturbances were applied to the wrists of 10 adult controls and 22 patients with essential tremor in order to study the interaction between mechanically-induced stretch-reflex oscillations and essential tremor. These two oscillations were separated by applying inertial and spring loads to the wrist. There was no evidence of increased or unstable stretch-reflex activity in the essential tremor patients, and stretch-reflex latencies did not correlate with the frequency of essential tremor. Essential tremor and mechanically-induced stretchreflex oscillations are separate phenomena capable of complex interaction.

The pathophysiological role of the stretch reflex in essential tremor remains unclear. Lee and Stein ${ }^{1}$ have shown that brief mechanical wrist disturbances produce variable resetting of the essential tremor rhythm, thus proving that the essential tremor oscillator receives peripheral sensory feedback. However, Marsden $e t a l^{2}$ applied sinusoidal torque disturbances to the fingers of essential tremor patients and found that essential tremor could not be entrained at frequencies differing by only $1.0 \mathrm{~Hz}$ from the spontaneous tremor frequency. This failure to entrain essential tremor reduces the importance of the stretch reflex in tremor genesis and supports the notion of a central oscillator mechanism.

Mechanical perturbations normally produce damped finger and wrist oscillations which are a direct result of the underdamped mechanical properties of these joints and controlling musculature. ${ }^{3-5}$ These oscillations are detected by peripheral sensory afferents and may thus induce reflex-evoked modulation of motor unit activity. ${ }^{5-6}$ This normal mechanical-reflex oscillation is expected to interact with essential tremor, and depending upon the strength of this interaction, one oscillation might greatly modify or entrain the other. ${ }^{78}$ Therefore, to identify potential abnormalities in the mechanicalreflex system, one should first try to separate the

Address for reprint requests: Dr R Elble, Department of Medicine, Southern Illinois University School of Medicine, P.O. Box 3926, Springfield, Illinois 62708, USA

Received 5 September 1986 and in revised form 16 November 1986. Accepted 22 November 1986 mechanical-reflex and essential tremor oscillations and then study the nature of their interaction. Separation of these two oscillations is possible because the frequency of essential tremor is not significantly altered by external spring and inertial loads while the frequency of the mechanical-reflex oscillation is changed in a manner predicted by second-order mechanics. ${ }^{910}$ Experiments of this type are now described.

\section{Methods}

Twenty-two patients with essential tremor and 10 agematched healthy adults (ages 25-70 yr) were studied after signing informed consent. All subjects were selected from a larger population of patients and controls that participated in a recently published companion study comparing physiological and essential tremor. ${ }^{9}$ The controls were equally divided into two groups: those with and those without prominent 8 - to $12-\mathrm{Hz}$ tremor components. The patients had tremor ranging from mild to severe and were free of medications known to affect tremor. Caffeinated beverages and tobacco were prohibited on the days of study. To avoid fatigue, the following studies were conducted in two experimental sessions lasting approximately 2 hours each.

Bipolar forearm electromyograms (EMGs) were recorded simultaneously from extensor and flexor muscles with $0.8 \mathrm{~cm}$ skin electrodes positioned longitudinally over the muscle, approximately $2 \mathrm{~cm}$ apart. Recordings were made from the extensor carpi radialis brevis, extensor digitorum communis, flexors carpi radialis and ulnaris, and palmaris longus.

The right hand, with fingers fully extended, was strapped to a plastic manipulandum attached directly to the shaft of a DC torque motor (Mavilor MT 300). The motor was mounted on a steel truss which also supported and restrained the horizontally positioned forearm. This apparatus thus restricted wrist movement to extension and flexion in the 
horizontal plane. The combined inertia of the manipulandum and torque motor drive shaft was $0.0024 \mathrm{~kg} \mathrm{~m}^{2}$. This is roughly equivalent to placing $240 \mathrm{~g}$ on the horizontally extended hand as described in our companion study. ${ }^{9}$ The motor was driven by a servo amplifier which was controlled by a Commodore VIC 20 computer and digital-to-analog converter. Wrist angle was measured with a precision potentiometer attached directly to the motor shaft. Acceleration was recorded using a miniature accelerometer attached to the manipulandum. The wrist angle and acceleration signals were high-pass filtered to remove DC content.

In one set of experiments, a series of $7050 \mathrm{~ms}$ torque pulses was applied to the wrist. The direction (extension versus flexion) of these perturbations was randomly varied using the VIC 20. The interval between pulses varied randomly between 2 and 12 seconds, and the torque pulse amplitudes were systematically varied from 0.25 to $4.0 \mathrm{Nm}$. When comparing the amplitudes of our torque perturbations with those of other authors (such as Lee and Stein $^{1}$ ), one must consider that a motor produces a torque which is attenuated by the manipulandum and mechanical load, and the actual torque delivered to the wrist is therefore considerably less. For example, Lee and Stein ${ }^{1}$ reported the use of $3.6 \mathrm{Nm}$ perturbations but did not specify the inertia of their motor and manipulandum. In our experiments, torque disturbances greater than $3.0 \mathrm{Nm}$ were intolerable to all but our strongest patients and were used only during spring or inertial loading (see below). We therefore suspect that the inertia of our manipulandum was considerably less than that of Lee and Stein. Our torque disturbances produced mean wrist excursions as great as $\pm 30^{\circ}$, and to prevent injury, mechanical stops were us $d$ to prevent wrist excursions greater than $\pm 50^{\circ}$. In all of these experiments, the torque amplitude was systematically increased to the limit tolerated by each subject, but for most subjects, 0.75 to $1.5 \mathrm{Nm}$ perturbations produced the most consistent mechanical-reflex response in the absence of additional loads.

Mild essential tremor was frequently more evident in the fingers than in the entire hand. Therefore, five controls and five mild essential tremor patients were also studied by applying torque pulse disturbances to the right third metacarpophalangeal (MP) joint. A much smaller manipulandum (inertia $0.0004 \mathrm{~kg} \mathrm{~m}^{2}$ ) was attached to the extended third digit, and wrist movement was restricted by the steel truss. This apparatus was frequently more suitable for studying the effects of mechanical perturbations on mild essential tremor and the 8 to $12 \mathrm{~Hz}$ component of physiological tremor, as recorded from the extensor digitorum communis.

In a second set of experiments, a continuous 70 second train of pseudorandom extension and flexion torque steps was delivered to the wrist as described by Dufresne et al. ${ }^{11}$ This torque train was structured to produce a uniform forcing from 0 to $50 \mathrm{~Hz}$, the frequency range of physiological interest. The frequency spectrum of this forcing was therefore statistically flat. The torque train amplitude was systematically varied from 0.25 to $4.0 \mathrm{Nm}$, but amplitudes greater than $1.0 \mathrm{Nm}$ were too strong unless applied during external loading.

During all perturbation experiments, the subjects were told to stiffen their wrist (or metacarpophalangeal joint) in the neutral position such that the joint returned passively to this angle following the perturbations. Accuracy of joint angle was not emphasised, and the subjects were specifically instructed not to react "voluntarily". After a few practice runs, each subject quickly found the minimum muscle activation required for approximate return of joint angle to the starting position. Thus, every effort was made to measure purely passive reflex responses. ${ }^{411}$

Each subject underwent several trials of random torque pulses and pseudorandom torque trains. These trials were performed with and without spring and inertial loads applied to the wrist. Inertial loads of 0.0045 and $0.0090 \mathrm{~kg}$ $\mathrm{m}^{2}$ were added by attaching 450 and $900 \mathrm{~g}$ respectively to the manipulandum. Spring loads were simulated by running feedback from the precision potentiometer to the motor servo amplifier. The natural frequency of the perturbationinduced wrist oscillations was directly proportional to the square root of the manipulandum stiffness (that is position feedback gain) and inversely proportional to the square root of the inertial load. ${ }^{910}$

Wrist angle, manipulandum acceleration and forearm EMGs were recorded simultaneously on an 8-channel instrument tape recorder. A series of code pulses, produced by the VIC 20, was also recorded and provided a permanent record of the precise timing and direction (extension versus flexion) of the torque perturbations. Prior to subsequent computer analysis, the EMG signals were fullwave rectified and lowpass filtered $(-3 \mathrm{db}$ at $30 \mathrm{~Hz})$ to produce the "integrated" (demodulated) EMG. ${ }^{912}$

The integrated EMG, wrist angle, and acceleration data acquired in the torque pulse experiments were analysed in the time domain by the methods of computed average transients (fig 1) ${ }^{13}$ Using a PDP $11 / 23$ computer, thirty $1 \cdot 5$-second epochs of these signals were simultaneously sampled, time locked to the perturbations. The sampling of each epoch began 0.3 seconds prior to each perturbation. The average wrist angle and EMG response to perturbation were therebs computed. Computer software was developed which allowe us to average these same data epochs on the essential tremor EMG burst preceding each perturbation and on any tremor burst following the perturbation. All perturbations were applied randomly with respect to the tremor cycle.

Short and medium latency reflex responses (M1 and $M 2$ of Lee and Tatton ${ }^{14}$ ) were obtained from the averaged integrated EMG responses to torque pulse perturbations with no external loading. The amplitudes of M1 and $\mathrm{M} 2$ (relative to the background EMG) were divided by the average background EMG level to produce "normalised" response amplitudes that could be compared across subjects. M1 and M2 onset and peak latencies were also measured. We found that reproducible reflex responses were obtained from all subjects with perturbations of 0.75 to $1.5 \mathrm{Nm}$ and that stronger perturbations did not alter reflex amplitude or latency. The reported $\mathrm{M} 1$ and $\mathrm{M} 2$ values were obtained with the optimum perturbation strength $(0 \cdot 75,1 \cdot 0$, or $1.5 \mathrm{Nm})$ for each subject. M1 and M2 amplitude and latency values did not differ among the muscles studied, and we therefore averaged our $\mathrm{M} 1$ and $\mathrm{M} 2$ values to obtain the generic values reported in this paper (mean $\pm S D$ ). Reflex latency and amplitude values for patients and controls were compared using both Student's $t$ test and Mann-Whitney rank-sum techniques. Similarly, both Pearson and Spearman correlation methods were used to look for relationships between (1) essential tremor frequency and reflex latencies and (2) tremor and reflex amplitudes. The null hypothesis (no difference or no 


\section{Time domain analysis}

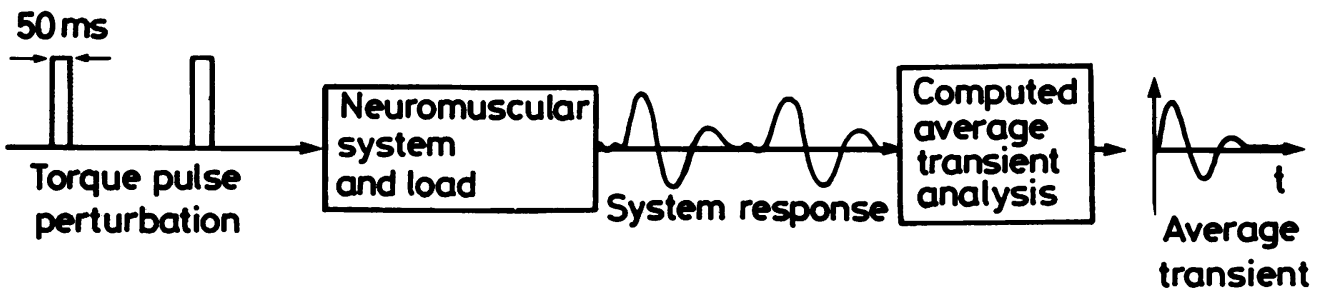

\section{Frequency domain analysis}

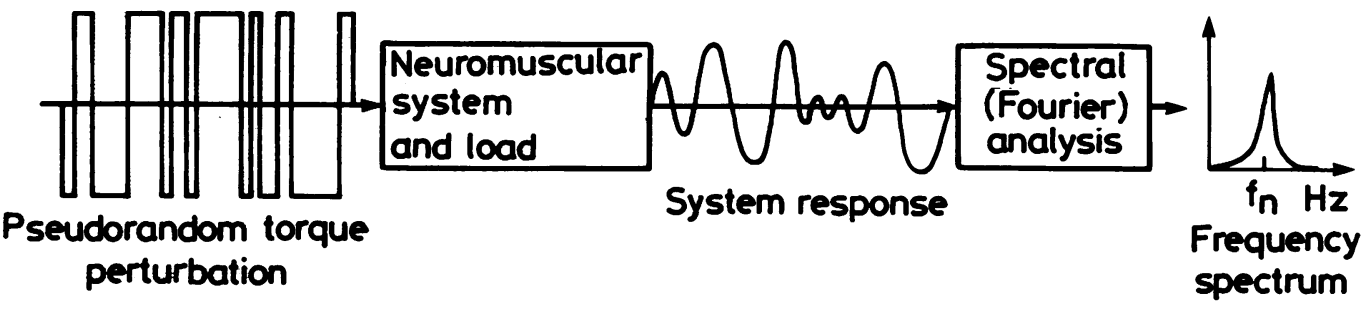

Fig 1 Flow diagram of the torque pulse and pseudorandom torque step experiments. In one set of experiments, $50 \mathrm{~ms}$ torque pulses were delivered randomly with respect to time and direction. Thirty $1.5 \mathrm{~s}$ epochs of wrist angle and integrated EMG were then averaged time-locked to the perturbations. The EMG and wrist angle responses to pseudorandom torque train forcing were analysed in the frequency domain, looking for preferred frequencies of oscillation $\left(f_{n}\right)$.

correlation) was rejected at $\mathrm{p}<0.05$.

The pseudorandom torque train data were analysed in the frequency domain using the PDP 11/23 (fig 1). Integrated EMG and wrist angle were analysed by auto- and crossspectral analysis as previously described, ${ }^{912} 15$ thus providing quantitative measurements of torque-induced wrist oscillation, reflex-evoked EMG modulation, and essential tremor EMG bursting. All spectra were smoothed to provide chi-squared spectral estimates with 56 degrees of freedom, and as a result of this smoothing, spectral peak-to-valley ratios of 2:1 are significant at $p<0.01 .^{15}$ Mathematically, the torque-pulse and torque-train forcings are identical and simply permit a "system response" analysis in the time domain and frequency domain, respectively (fig 1).

\section{Results}

\section{Single Torque Pulse Experiments}

Figure 2 illustrates the averaged integrated EMG and wrist angle response to 30 extensor torque pulses for a control subject with a prominent $8-$ to $12-\mathrm{Hz}$ tremor component. The initial wrist excursion produced M1 and $M 2$ reflex responses in the integrated EMG, followed by one or more damped oscillations in wrist angle. The frequency of this mechanical-reflex response was $5-7 \mathrm{~Hz}$ in all subjects (fig $2 \mathrm{~A}$ ). This is precisely the frequency of the mechanical-reflex component of physiological tremor when the unrestricted hand is extended horizontally with an external inertial load equivalent to that of the manipulandum and torque motor. ${ }^{9}$ Additional inertial loads reduced the frequency of these oscillations to $3-4 \mathrm{~Hz}$ (fig 2C), and spring loading increased the frequency to $9-10 \mathrm{~Hz}$ (fig 2B). Attempts to increase the frequency above this range were not successful owing to mechanical and electronic limitations of the torque motor and servo amplifier.

In addition to this normal mechanical-reflex oscillation, patients with essential tremor exhibited rhythmic bursts of EMG activity at mean frequencies ranging from 5 to $12 \mathrm{~Hz}$. For a given patient, the mean frequency of these bursts was invariant, in direct contrast to that of the mechanical-reflex oscillation. Visual analysis of a single data epoch often gave the false impression that the essential tremor bursts were time-locked to the perturbation (fig 3B). However, computer averages of 30 or more epochs revealed that only the damped mechanical-reflex EMG oscillation was generated time-locked to the perturbations (fig 3A). The presence of the essential tremor rhythm before the perturbations was readily demonstrated by averaging the same data epochs on the tremor burst preceding each perturbation (fig 3C). Similarly, resumption of the tremor rhythm following the perturbations was demonstrated by averaging the data epochs on the last tremor burst of each epoch (fig 3D). Note that while the essential tremor rhythm was transiently disrupted by the perturbations (fig $3 \mathrm{C}$ and D), it was not reset time-locked to the perturbations 

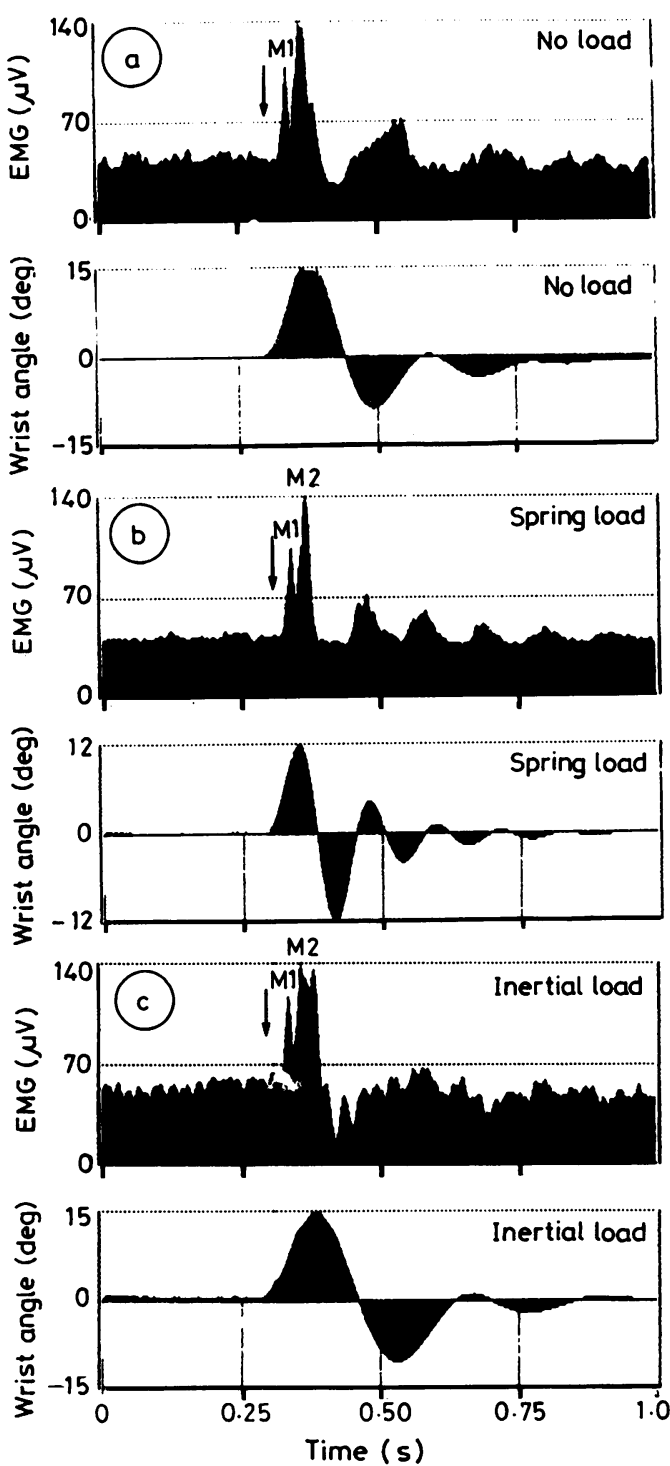

Fig 2 The $50 \mathrm{~ms}$ torque pulses invariably produced damped wrist oscillation ( $5 \cdot 3 \mathrm{~Hz}$; wrist flexion is positive) which was associated with damped reflex modulation of EMG (integrated EMG in this and all other figures). Inertial loading $(c)$ reduced the frequency of this oscillation $(3.6 \mathrm{~Hz})$ while spring loading $(b)$ increased it $(9.0 \mathrm{~Hz})$. The prominent 8- to $12-\mathrm{Hz}$ tremor component in this subject was not seen in these averages because it was not reset time-locked to the perturbations $(1.5 \mathrm{Nm}$ in a, $3.0 \mathrm{Nm}$ in $b$, and $2.0 \mathrm{Nm}$ in $\mathrm{c}$ ).

(fig 3A). Hence, the reflex response and essential tremor bursts are two separate phenomena.

The 8- to $12-\mathrm{Hz}$ component of physiological tremor interacted with the mechanical-reflex oscillations in a manner qualitatively identical to high-frequency essential tremor illustrated in fig 3 . There was always a well-defined mechanical-reflex response in addition to the 8- to $12-\mathrm{Hz}$ bursts of EMG, and this 8- to $12-\mathrm{Hz}$ activity was disrupted but not reset by the perturbations.

In patients with advanced essential tremor, the mechanically-induced EMG modulation was not as clean as in the controls (fig 4), and in patients with greatest tremor, this EMG modulation was evident only during periods of waning tremor amplitude, regardless of perturbation strength. Although advanced essential tremor could be disrupted by the largest perturbations, we observed no consistent timelocked resetting (fig 4).

The absence of consistent essential tremor resetting was demonstrable in all patients studied. However, in

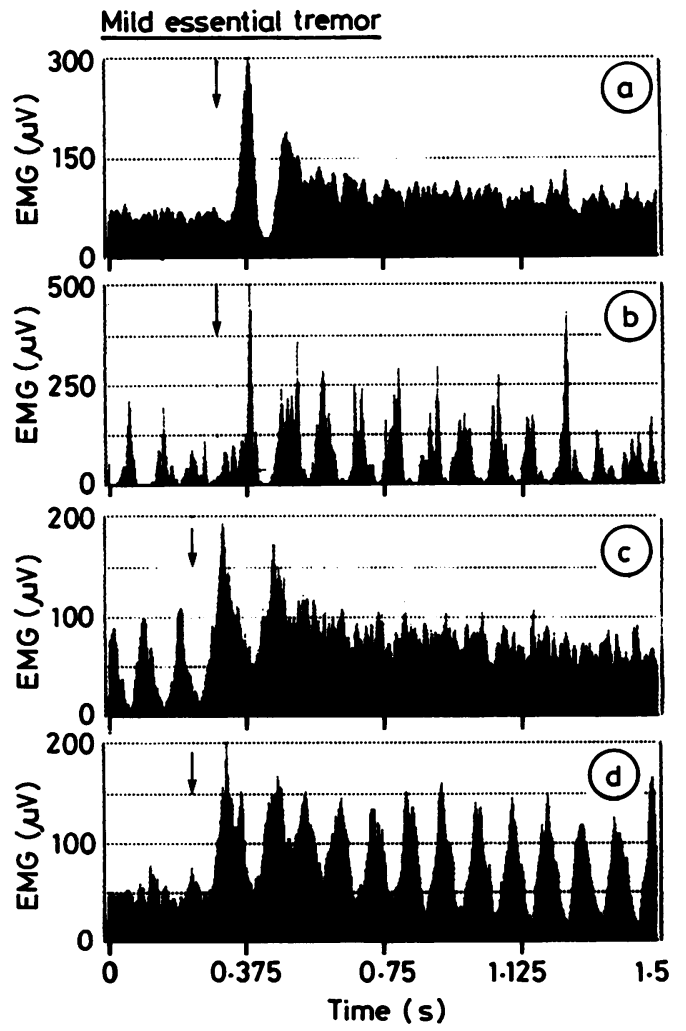

Fig 3 Integrated extensor digitorum communis EMG response to metacarpophalangeal joint perturbation in a patient with mild familial tremor. The 8- to $12-\mathrm{Hz}$ EMG bursts seen in single data epochs (b) were not evident in the average of 30 epochs (a) time-locked to the perturbations (0.25 Nm, arrow). The 8- to $12-\mathrm{Hz}$ rhythm was evident when the same 30 epochs were averaged time-locked to the EMG burst preceding each perturbation (c) or time-locked tó the last tremor burst of each epoch $(d)$. 
Advanced essential tremor

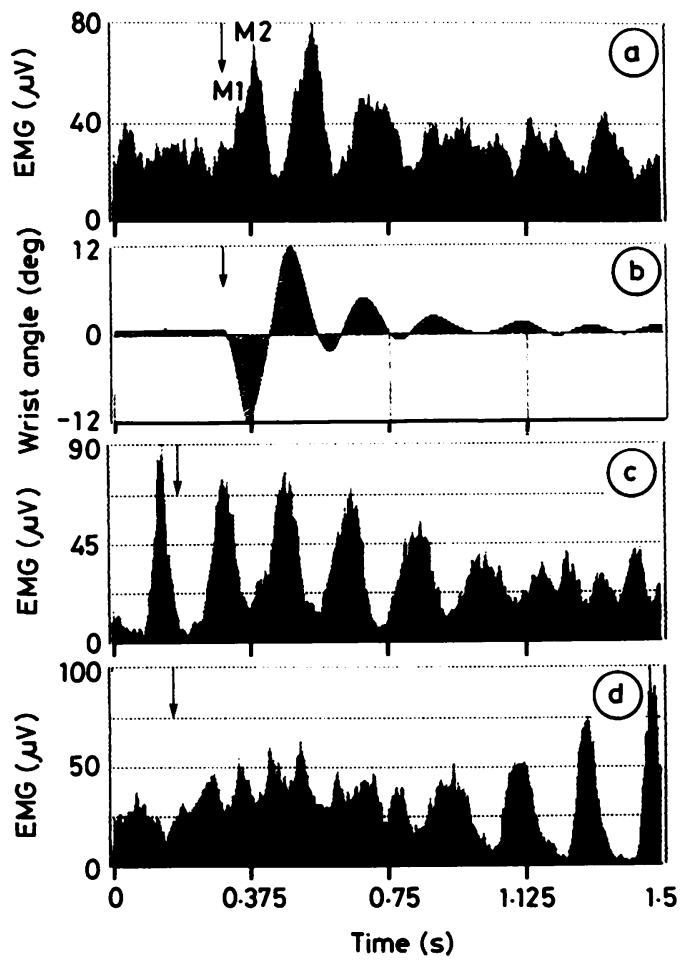

Fig 4 In patients with advanced essential tremor, the reflex modulation of EMG was much less clean (A) than it. the milder patients (fig 3A) and controls (fig 2). By averaging the same thirty epochs on the essential tremor burst preceding each perturbation $(1.5 \mathrm{Nm})$, one could see that the rhythm $(5.6 \mathrm{~Hz})$ was relatively undisturbed $(C)$. In $D$, the same data were averaged on the last tremor burst of each epoch to illustrate the resumption of essential tremor following the period of mechanical-reflex oscillation $(4.6 \mathrm{~Hz})$.

the course of our studies, it soon became apparent that the results of our perturbation experiments depended upon several factors. First, mass loading frequently produced a dramatic suppression of the essential tremor rhythm, a phenomenon previously observed by Hewer et al. ${ }^{16}$ The degree of suppression waxed and waned, but most subjects exhibited sufficient periods of waning suppression to permit adequate data collection. Second, the absence of essential tremor resetting was most demonstrable when the inertial and/or spring loads were adjusted to create a frequency mismatch between the mechanicalreflex and essential tremor oscillations. When these loads were adjusted to match the frequencies, variable resetting, similar to that of Lee and Stein, ${ }^{1}$ was observed with perturbations of $0.75 \mathrm{Nm}$ or greater.
However, it was impossible to tell whether this was true resetting or simply the essential tremor rhythm being overwhelmed by large mechanical-reflex oscillations at the same frequency. Indeed, the absence of resetting was most evident in patients with greatest essential tremor. During periods of greatest tremor, it was often impossible to produce a consistent reflex response, regardless of mechanical-reflex frequency. Instead, these patients exhibited a variably damped wrist oscillation with no associated EMG modulation, and the ongoing essential tremor rhythm (EMG) seemed impervious to the perturbations.

There was no correlation between essential tremor frequency and the latencies of $M 1$ and $M 2$, and the mean onset and peak latencies of M1 (onset $=26 \pm$ $4 \mathrm{~ms}$; peak $=39 \pm 6 \mathrm{~ms}$ ) and M2 (onset $=48 \pm 6 \mathrm{~ms}$; peak $=71 \pm 10 \mathrm{~ms}$ ) did not differ from control values (M1 onset: peak $=26 \pm 4: 38 \pm 6 \mathrm{~ms}$; $\mathrm{M} 2$ onset: peak $=48 \pm 6: 71 \pm 7 \mathrm{~ms}$ ). Similarly, there was no correlation between tremor amplitude and the normalised amplitudes of M1 $(0.69 \pm 0.21)$ and M2 $(1.95 \pm 1.3)$ which likewise did not differ from control values $(0.92 \pm 0.44$ and $1.98 \pm 1.5$, respectively).

\section{Pseudorandom torque step experiments}

In all subjects, the pseudorandom torque trains produced a resonant mechanical wrist oscillation at 5-7 Hz. This oscillation corresponded to the underdamped mechanical-reflex oscillation produced in the single torque pulse experiments. Normal subjects exhibited a prominent EMG spectral peak produced by the mechanical-reflex modulation of motor units (fig 5). Inertial loads decreased the frequency of these oscillations (fig 5, arrow) while spring loads increased frequency (not shown). Controls with prominent 8- to $12-\mathrm{Hz}$ tremor exhibited a second spectral peak in the EMG spectrum (fig 5). The frequency of this oscillation did not change with inertial loading (fig 5) and is therefore similar to high-frequency essential tremor (fig 6).

In patients with essential tremor, the mechanicallyinduced EMG modulation was less prominent relative to the frequency-invariant essential tremor bursts, and consequently, the essential tremor spectral peak in the integrated EMG spectrum was proportionally larger than the mechanical-reflex peak (fig 6). In the most advanced patients, only a single EMG spectral peak, at the frequency of essential tremor, could be discerned during the periods of greatest tremor (fig 6). Thus, waxing essential tremor appeared to prevent normal mechanical-reflex modulation of motor unit activity, regardless of perturbation strength.

\section{Discussion}

We have demonstrated in both controls and essential 


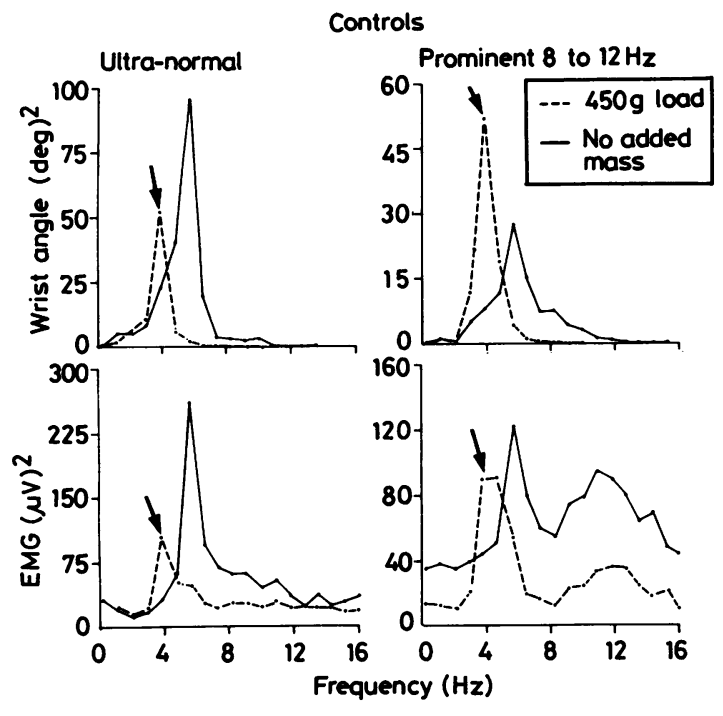

Fig 5 Pseudorandom torque step data $(0.5 \mathrm{Nm})$. "Ultranormal" controls (with no 8 to $12 \mathrm{~Hz}$ tremor) typically exhibited a prominent integrated EMG spectral peak $(6 \mathrm{~Hz})$ produced by mechanical-reflex modulation of motor units. Inertial loads decreased the frequency of these oscillations ( $4 \mathrm{~Hz}$, arrows). Controls with prominent 8 to $12 \mathrm{~Hz}$ tremor components exhibited a corresponding peak in the EMG spectrum $(11 \mathrm{~Hz})$ in addition to the mechanicalreflex peak.

tremor patients that mechanical perturbations produce wrist oscillations which result from underdamped mechanical properties and which therefore have a frequency that is a function of spring and inertial loading. When these oscillations reach sufficient amplitude, modulation of motor unit activity occurs through the stretch reflex. ${ }^{6}$ In our torque pulse experiments, the essential tremor rhythm and the 8- to $12-\mathrm{Hz}$ component of physiological tremor were disrupted by random perturbations but were not consistently reset in a time-locked fashion. In addition, the frequencies of these oscillations were not correlated with the short and intermediate reflex latencies (M1 and M2). The mechanical-reflex EMG modulation, by contrast, was time-locked to the perturbations, as one would expect of any oscillation generated in the peripheral stretch reflex. ${ }^{7}$ We conclude that while the 8- to $12-\mathrm{Hz}$ and essential tremor oscillators may receive sensory feedback, these oscillations are distinct from the normal mechanical-reflex oscillation, present in all subjects.

Lippold reported that finger perturbations reset the 8- to $12-\mathrm{Hz}$ component of physiological tremor and therefore argued that this oscillation is due to stretch reflex instability. ${ }^{17}$ However, in his initial publication, computer averaging techniques were not used to determine if this resetting was consistently time-locked to the perturbations. ${ }^{17}$ In addition, his extensor digitorum EMG records (fig 10 in reference 17 ) reveal that the 8- to $12-\mathrm{Hz}$ bursts of motor unit activity began more than $100 \mathrm{~ms}$ after the perturbation, suggesting that this activity may represent a triggered reaction to the stimulus as opposed to a time-locked reflex oscillation. In a subsequent paper, Gottlieb and Lippold ${ }^{18}$ used computer averaging techniques to analyse similar data, and time-locked resetting of the $8-$ to $12-\mathrm{Hz}$ rhythm was not convincingly demonstrated (see their fig 4 and compare with our fig 3 ). Therefore, while we do not find Lippold's data significantly dissimilar from ours, we differ greatly in the interpretation of these data, finding no reason to invoke stretch reflex instability.

A prominent 8- to $12-\mathrm{Hz}$ tremor can be demonstrated only in a minority of controls, ${ }^{9,18}$ and aside from being lower in amplitude, this tremor is indistinguishable from high-frequency essential tremor. ${ }^{9}$ Several studies have shown that this $8-$ to $12-\mathrm{Hz}$ tremor is not an inevitable product of normal stretch reflex function, ${ }^{19-22}$ a conclusion supported by our data from ultranormal controls (fig 5). In this regard, we would emphasize that previous investigators did

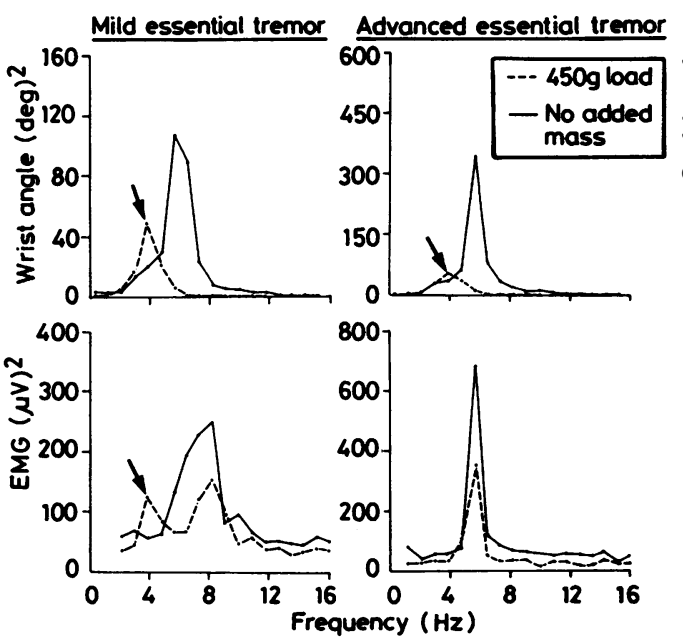

Fig 6 In patients with mild essential tremor, the pseudorandom forcing $(0.5 \mathrm{Nm})$ produced a $6-\mathrm{Hz}$ wrist oscillation which decreased to $4 \mathrm{~Hz}$ with $450 \mathrm{~g}$ added mass, and there was a corresponding reflex modulation of EMG (arrow) which was distinct from the frequency-invariant EMG oscillation produced by essential tremor $(8 \mathrm{~Hz})$. In advanced patients, inertial loading reduced the frequency of the wrist oscillations ( $4 \mathrm{~Hz}$, arrow), but there was no corresponding mechanical-reflex EMG peak. Only a single finely-tuned essential tremor peak could be identified in the EMG spectrum $(5.5 \mathrm{~Hz})$. 
not report 8- to $12-\mathrm{Hz}$ oscillations following wrist ${ }^{56}$ and finger ${ }^{3}$ perturbations in adult controls. Furthermore, most normal subjects exhibit little or no evidence of motor unit synchronisation except during fatigue, ${ }^{92324}$ and the activated ("enhanced") physiological tremor typically seen in fatigue is the mechanical-reflex oscillation, not the $8-$ to $12-\mathrm{Hz}$ tremor. 61019

Because the frequency of the $8-$ to $12-\mathrm{Hz}$ and essential tremors is independent of peripheral mechanical factors and reflex latencies, a central oscillator probably plays a key mechanistic role. Our study and others ${ }^{12}$ indicate that the central oscillator(s) responsible for these tremors are coupled to the stretch reflex. The stretch reflex may therefore play a role in governing the amplitude of these oscillations, but it does not produce the essential tremor rhythm. Furthermore, the interaction between these coupled oscillators may be synergistic or competitive, depending upon the experimental conditions. ${ }^{7-8}$ Basic oscillator theory tells us that this interaction will depend upon (1) the relative strengths of the two oscillations, (2) the relative frequencies of the two oscillations, and (3) the strength of coupling between the two oscillations. ${ }^{25}$

Our hypothesis of coupled stretch reflex and central oscillators explains the apparent conflicts between the work of Lee and Stein ${ }^{1}$ and Marsden et al. ${ }^{2}$ In experiments very similar to ours, Lee and Stein ${ }^{1}$ reported variable resetting of advanced essential tremor but did not separate the mechanical-reflex and essential tremor oscillations. Strong perturbations to these mutually-entrained oscillators would be expected to produce the high degree of time-locked resetting observed in their experiments. Similarly, we observed greatest essential tremor resetting when two conditions were met: (1) the mechanical-reflex oscillation was much larger than the ongoing essential tremor and (2) there was no frequency mismatch between the two oscillations. During a frequency mismatch, however, the two oscillations were clearly competitive, with the stronger oscillation tending to disrupt and obscure the other.

Lee and Stein did not find complete resetting in any of their patients and therefore emphasised that a central oscillator may play a role in tremor genesis. Such a role is also indicated by the work of Marsden et $a l^{2}{ }^{2}$ who demonstrated that essential tremor has a preferred frequency and cannot be entrained by mechanically-imposed reflex oscillations at a slightly different frequency. In effect, Marsden et al imposed a frequency mismatch between essential tremor and mechanical-reflex oscillators, thus testing their relative strengths and coupling. Instead of entrainment, they observed predictable beats in the essential tremor rhythm. ${ }^{2}$ The experiments of Marsden et $a l^{2}$ are analogous to our torque train studies with inertial loading in advanced tremor patients. Resonant mechanical wrist oscillations at frequencies above and below the frequency of essential tremor produced no entrainment of essential tremor (fig 6).

Essential tremor and normal mechanical-reflex tremor have distinctly different EMG/wrist oscillation amplitude relationships which are consistent with the mechanisms we have proposed. The mechanical-reflex wrist oscillation is primarily the result of underdamped limb mechanics, and its EMG modulation is in response to this oscillation rather than its direct cause. Unless this oscillation is accentuated by fatigue (increased reflex gain?) or external perturbations, there is little discernible EMG modulation. Consequently, mechanical-reflex tremor is usually large relative to its EMG. By contrast, the wrist tremor of any central oscillator will be proportionally smaller relative to its EMG. This is because a central oscillator produces tremor directly by rhythmic EMG modulation. However, the effect of this modulation is minimised by the attenuating properties of skeletal muscle, limb mechanics and external loads. Therefore, prominent essential tremor and 8- to $12-\mathrm{Hz}$ physiologic tremor are commonly detectable in the EMG with little discernible wrist oscillation, precisely the opposite relationship observed for mechanical-reflex tremor (figs 5 and 6, and figs 1-3 in reference 9).

Tremor research is historically riddled with heated controversy regarding the relative roles played by stretch-reflex and central oscillators. Because an underdamped mechanical-reflex system is present in everyone, the possibility of coupled oscillators must be considered in the study of any tremor. Theoretical studies by Stein and coworkers ${ }^{7-8}$ have vividly illustrated the complexities which may result from such oscillator interaction, and our study as well as those of Lee and Stein ${ }^{1}$ and of Marsden et al ${ }^{2}$ support the relevance of coupled oscillator theory in any discussion of essential tremor. We would also argue that the coupled oscillator model applies to the mechanical-reflex and $8-$ to $12-\mathrm{Hz}$ components of physiological tremor.

Supported by grants from the National Institute of Neurological and Communicative Disorders and Stroke (RO1-NS20973) and from the American Parkinson Disease Association.

Presented in part at the thirty-eighth annual meeting of the American Academy of Neurology, New Orleans, LA, and at the fifteenth annual meeting of the Society for Neuroscience, Dallas TX.

The authors are indebted to James Adelman and John Holcomb for their technical assistance and to Rita Cerar for typing this manuscript. 


\section{References}

1 Lee RG, Stein RB. Resetting of tremor by mechanical perturbations: a comparison of essential tremor and parkinsonian tremor. Ann Neurol 1981;10:523-31.

2 Marsden CD, Obeso JA, Rothwell JC. Benign essential tremor is not a single entity. In: Yahr MD, ed. Current Concepts of Parkinson Disease and Related Disorders, Amsterdam: Excerpta Medica, 1981:31-46.

3 Kelso JAS, Holt KG. Exploring a vibratory systems analysis of human movement production. $J$ Neurophysiol 1980;43:1183-96.

4 Neilson PD, Neilson MD. The role of action reflexes in the damping of mechanical oscillations. Brain Res 1978; 142:439-53.

5 Stiles RN. Lightly damped hand oscillations: acceleration-related feedback and system damping. $J$ Neurophysiol 1983;50:327-43.

6 Hagbarth K-E, Young RR. Participation of the stretch reflex in human physiological tremor. Brain 1979;102:509-26.

7 Stein RB, Gordon T, Oguztoreli MN, Lee RG. Classifying sensory patterns and their effects on locomotion and tremor. Can J Physiol Pharmacol 1981;59:645-55.

8 Stein RB, Lee RG. Tremor and clonus. In: Brooks V, ed. Handbook of Physiology, Motor Control, Baltimore: Williams and Wilkins, 1981:325-43.

9 Elble RJ. Physiologic and essential tremor. Neurology 1986;36:225-31.

10 Elble RJ, Randall JE. Mechanistic components of normal hand tremor. Electroencephalogr Clin Neurophysiol 1978;44:72-82.

11 Dufresne JR, Soechting JF, Terzuolo CA. Reflex motor output to torque pulses in man: identification of shortand long-latency loops with individual feedback parameters. Neurosci 1979;4:1493-1500.

12 Elble RJ, Randall JE. Motor-unit activity responsible for the 8- to $12-\mathrm{Hz}$ component of human physiological finger tremor. $J$ Neurophysiol 1976;39:370-83.
13 Glaser EM, Ruchkin DS. Principles of Neurobiologic Signal Analysis. New York: Academic Press, 1976:177-290.

14 Lee RG, Tatton WG. Motor responses to sudden limb displacement in primates with specific CNS lesions and in human patients with motor system disorders. Can J Neurol Sci 1975;2:205-93.

15 Jenkins GM, Watts DG. Spectral Analysis and Its Applications. San Francisco: Holden-Day, 1968:209-57.

16 Hewer RL, Cooper R, Morgan MH. An investigation into the value of treating intention tremor by weighting the affected limb. Brain 1972;95:579-90.

17 Lippold OCJ. Oscillation in the stretch reflex arc and the rhythmical $8-12 \mathrm{c} / \mathrm{s}$ component of physiological tremor. J Physiol (Lond) 1970;206:359-82.

18 Gottlieb S, Lippold OCJ. The 4-6 Hz tremor during sustained contraction in normal human subjects. J Physiol (Lond) 1983;336:499-509.

19 Stiles RN. Mechanical and neural feedback factors in postural hand tremor of normal subjects. $J$ Neurophysiol 1980;44:40-9.

20 Andrews CJ, Burke D, Lance JW. The comparison of tremors in normal, parkinsonian and athetotic man. J Neurol Sci 1973;19:53-61.

21 Brown TIH, Rack PMH, Ross HF. Different types of tremor in the human thumb. $J$ Physiol (Lond) 1982;332:113-23.

22 Marsden CD, Meadows JC, Lange GW, Watson RSO Effect of deafferentation on human physiologica历 tremor. Lancet 1967;2:700-2.

23 Dietz V., Bischofberger E, Witz C, Freund H-J. Cor尺 relation between the discharges of two simultaneousl ${ }^{2}$ recorded motor units and physiological tremor. Elec troencephalogr Clin Neurophysiol 1976;40:97-105.

24 Freund H-J. Motor unit and muscle activity in voluntary. motor control. Physiol Rev 1983;63:387-436.

25 McMahon TA. Muscles, Reflexes, and Locomotion: Princeton, NJ: Princeton University Press, 1984:174-80 